\title{
LINK-A IncRNA participates in the pathogenesis of glioma by interacting with survivin
}

\author{
XIA HUA, GUANGXING LI, ZHONGTAO LIU and ZHANFENG NIU \\ Department of Neurosurgery, General Hospital of Ningxia Medical University, Yinchuan, Ningxia 750003, P.R. China
}

Received September 12, 2018; Accepted May 3, 2019

DOI: $10.3892 / \mathrm{etm} .2019 .7716$

\begin{abstract}
Long intergenic non-coding RNA for kinase activation (LINK-A) long non-coding RNA (lncRNA) has been characterized in triple negative breast cancer, but its potential involvement in glioma has not been investigated. In the present study, serum levels of LINK-A lncRNA and survivin in patients with glioma and healthy controls were determined by RT-qPCR and ELISA, respectively. The diagnostic value of serum LINK-A IncRNA for glioma was evaluated by receiver operating characteristic (ROC) curve analysis. Potential correlations between serum levels of LINK-A lncRNA and survivin were analyzed by Pearson correlation coefficient. LINK-A lncRNA siRNA, LINK-A IncRNA-carrying expression vector and survivin-carrying expression vector were transfected into glioma cells, and the effects on LINK-A IncRNA expression, survivin expression and cell apoptosis were explored by RT-qPCR, western blot analysis and annexin $\mathrm{V} /$ propidium iodide staining. It was observed that the serum levels of LINK-A lncRNA and survivin were significantly higher in patients with glioma compared with healthy controls. Increased levels of LINK-A IncRNA distinguished glioma patients from healthy controls, based on ROC curve analysis. Serum levels of LINK-A lncRNA and survivin were positively correlated in glioma patients, but not in healthy controls. Overexpression of LINK-A lncRNA led to increased survivin protein expression, while survivin overexpression had no effect on LINK-A lncRNA expression. LINK-A lncRNA and survivin overexpression each reduced glioma cell apoptosis, but LINK-A IncRNA siRNA-mediated knockdown increased apoptosis. Survivin overexpression attenuated the inducing effects of LINK-A lncRNA knockdown on apoptosis. In conclusion, LINK-A IncRNA inhibited glioma cell apoptosis potentially by the upregulation of survivin. The present study revealed that LINK-A may serve as possible diagnostic marker for glioma.
\end{abstract}

Correspondence to: Dr Xia Hua, Department of Neurosurgery, General Hospital of Ningxia Medical University, 804 Shengli Street, Xingqing, Yinchuan, Ningxia 750003, P.R. China

E-mail: gwcpv69@163.com

Key words: glioma, LINK-A lncRNA, survivin, apoptosis

\section{Introduction}

As a type of human malignancy originating from the glial cells of the spine or the brain, glioma accounts for $>30 \%$ of cases of central nervous system and brain tumors and $>80 \%$ of all malignant brain tumors $(1,2)$. Patients with brain glioma usually show headaches, seizures, cranial nerve disorders and vomiting, which are caused by the increased intracranial pressure. By contrast, weakness, pain and numbness in the extremities are the main symptoms of optic nerve glioma (3). Hereditary genetic disorders and activation of certain oncogenes have been associated with the development of gliomas $(4,5)$. At present, the molecular mechanism of the pathogenesis of glioma remains unclear, leading to poor treatment outcomes for patients with glioma.

Survivin, also known as baculoviral inhibitor of apoptosis repeat-containing 5 (BIRC5), is a member of the inhibitor of apoptosis family that inhibits programmed cell death or apoptosis through the inhibition of caspase activation (6). A growing body of literature has shown that survivin is frequently overexpressed in human cancers, and the overexpression of survivin inhibits cancer cell apoptosis $(7,8)$. At present, inhibition of survivin is reported to be a promising therapeutic target for cancer treatment $(9,10)$. It has been reported that the expression and degradation of survivin in cancer development can be regulated by long non-coding RNAs (lncRNAs) $(11,12)$. Long intergenic non-coding RNA for kinase activation (LINK-A) lncRNA has been characterized as an oncogenic lncRNA in triple negative breast cancer (13). In breast cancer, LINK-A IncRNA promotes cancer development by activating normoxic hypoxia-inducible factor 1- $\alpha$ (HIFl $\alpha$ ) signaling (13). Based on current knowledge, the involvement of LINK-A lncRNA in other human diseases is unknown. In addition, the interaction between LINK-A and survivin is still unknown. In the present study, the role of LINK-A IncRNA in the regulation of survivin expression and glioma cell apoptosis was investigated.

\section{Materials and methods}

Cell lines and human specimens. Blood was extracted from 52 patients with glioma and 38 healthy volunteers to prepare serum. The participants were admitted to the General Hospital of Ningxia Medical University (Yinchuan, China) between May 2015 and May 2018. The inclusion criteria for patients were: i) Diagnosis by histopathological examination; ii) no other 
severe diseases diagnosed; and iii) patients who understood the experimental protocol and signed informed consent. The exclusion criteria were as follows: i) Diagnosis with multiple diseases; and ii) patients who were treated within the 3 months before blood extraction. The 52 patients with glioma included 30 males and 22 females, with a mean age of $45.8 \pm 5.6$ years (range, 32-64 years). There were 12 cases of grade I, 13 cases of grade II, 10 cases of grade III and 17 cases of grade IV. The 38 healthy volunteers included 21 males and 17 females with a mean age of 47.1 \pm 5.4 years (range, 33-67 years). Patient and control groups showed similar age and sex distributions. The present study was approved by the Ethics Committee of the General Hospital of Ningxia Medical University. All patients and healthy volunteers signed written informed consent.

Human glioma cell lines CCD-25Lu and Hs 683 were provided by American Type Culture Collection (ATCC). ATCC-formulated Eagle's Minimum Essential Medium (cat. no. 30-2003) containing 10\% fetal bovine serum (ATCC) was used to culture cells of both cell lines at $37^{\circ} \mathrm{C}$ with $5 \%$ $\mathrm{CO}_{2}$ and $95 \%$ humidity.

ELISA. Human Survivin Quantikine ELISA kit (DSV00; R\&D Systems China Co., Ltd.) was used to measure levels of survivin in serum from patients with glioma and healthy controls. The assay was performed according to the manufacturer's protocol. Serum levels of survivin were expressed as $\mathrm{pg} / \mathrm{ml}$.

Reverse transcription quantitative PCR (RT-qPCR). RNA extractions were performed using GenElute ${ }^{\mathrm{TM}}$ Plasma/Serum RNA Purification Mini kit (RNB500-50RXN; Sigma-Aldrich; Merck KGaA) according to the manufacturer's protocol. Next, cDNA was synthesized using RevertAid RT Reverse Transcription kit (Thermo Fisher Scientific, Inc.). Samples were prepared for PCR using SYBR ${ }^{\mathrm{TM}}$ Green PCR Master Mix (Applied Biosystems; Thermo Fisher Scientific, Inc.). Thermocycling conditions for qPCR were as follows: $1 \mathrm{~min}$ at $95^{\circ} \mathrm{C}$, followed by 40 cycles of $10 \mathrm{sec}$ at $95^{\circ} \mathrm{C}$ and $40 \mathrm{sec}$ at $58^{\circ} \mathrm{C}$. Primers were as follows: LINK-A forward, 5'-TTC CCCCATTTTTCCTTTTC-3' and reverse, 5'-CTCTGGTTG GGTGACTGGTT-3'; $\beta$-actin forward, 5'-GACCTCTATGCC AACACAGT-3' and reverse, 5'-AGTACTTGCGCTCAGGAG GA-3'; and survivin forward, 5'-AAGAACTGGCCCTTCTTG GA-3' and reverse, 5'-CAACCGGACGAATGCTTTT-3'. The $2^{-\Delta \Delta \mathrm{Cq}}$ method (14) was used to quantify relative RNA levels and $\beta$-actin was used as the reference gene.

Vectors, siRNAs and cell transfection. SiRNA (5'-UCCACA CACCGCCUCCCACCU-3') targeting LINK-A IncRNA, scrambled negative control siRNA (5'-UUCUCCGAACGU GUCACGUdTdT-3'), LINK-A lncRNA expression vectors and survivin expression vectors were designed and synthesized by Shanghai GenePharma Co., Ltd. using pcDNA3.1 vector. Cell transfections were performed using Lipofectamine ${ }^{\mathrm{TM}} 2000$ transfection reagent (Invitrogen; Thermo Fisher Scientific, Inc.). The concentration of vector used for transfection was $15 \mathrm{nM}$ and the concentration of siRNAs was $45 \mathrm{nM}$. Cells treated with transfection reagent only, without siRNA or vectors, were control cells. Cells transfected with scrambled negative control siRNA or empty vectors were negative control cells. At 24 after transfection, expression of LINK-A lncRNA and survivin was detected by RT-qPCR to confirm that expression was increased to $>200 \%$ or decreased to $<50 \%$ prior to subsequent experiments (data not shown).

Cell apoptosis assay. Cells were harvested at $24 \mathrm{~h}$ after transfection. Cells with different treatments (LINK-A and survivin expression vector transfection groups, LINK-A siRNA transfection group, as well as corresponding control and negative control groups) aforementioned were harvested to make single cell suspensions $\left(6 \times 10^{4}\right.$ cells $\left./ \mathrm{ml}\right)$ using serum-free cell culture medium. Each well of a 6 -well plate was filled with $10 \mathrm{ml}$ cell suspension. Cells were cultivated under normal conditions for $48 \mathrm{~h}$. The cells were then subjected to trypsin $(0.25 \%)$ digestion. After staining with Annexin V-fluorescein isothiocyanate (Dojindo Molecular Technologies, Inc.) and propidium iodide (PI; Dojindo Molecular Technologies, Inc.), apoptotic cells were detected by flow cytometry. Cyflogic free flow cytometry software version 1.2.1 (http://www.cyflogic. $\mathrm{com} /$ ) was used to analyze data.

Western blot analysis. Total Protein Extraction (TPE $\left.{ }^{\mathrm{TM}}\right) \mathrm{kit}$ (VWR International Co.) was used for the extraction of total protein from samples. A BCA kit (Sangon Biotech Co., Ltd.) was used to measure protein concentrations. After denaturing, protein samples were separated by SDS-PAGE using a $10 \%$ gel with $20 \mu \mathrm{g}$ per lane. Following transfer to PVDF membranes, blocking was performed in PBS containing 5\% fat-free milk at room temperature for $2 \mathrm{~h}$. The membranes were then incubated with primary antibodies of Rabbit anti-human survivin (1:1,400; ab76424; Abcam) and rabbit anti-human GAPDH (1:1,400; ab9485; Abcam) overnight at $4^{\circ} \mathrm{C}$. Following this, membranes were then incubated with goat anti-rabbit IgG-horseradish peroxidase secondary antibody $(1: 1,300$; MBS435036; MyBioSource, Inc.) at $37^{\circ} \mathrm{C}$ for $2 \mathrm{~h}$. Pierce ECL western blotting substrate (Thermo Fisher Scientific, Inc.) was used to develop signals. MYECL ${ }^{\mathrm{TM}}$ Imager (Thermo Fisher Scientific, Inc.) was used to scan signals and ImageJ version 1.46 software (National Institutes of Health) was used to quantify band intensity.

Statistical analysis. Experiments were repeated three times. Data are presented as mean \pm standard deviation and Graphpad Prism 6 software (GraphPad Software, Inc.) was used for all statistical analyses. Diagnostic analysis was performed by receiver operating characteristic (ROC) curve analysis with glioma patients as true positive cases and healthy controls as true negative cases. Correlation between serum levels of LINK-A lncRNA and survivin was analyzed using Pearson's correlation coefficient. Comparisons were performed using Student's t-test when comparing two groups and one-way analysis of variance followed by Tukey test when comparing more than two groups. $\mathrm{P}<0.05$ was considered to indicate a statistically significant difference.

\section{Results}

Serum levels of LINK-A IncRNA and survivin are significantly higher in patients with glioma compared with healthy controls. RT-qPCR results showed that, compared with healthy controls, serum levels of LINK-A lncRNA were significantly increased 

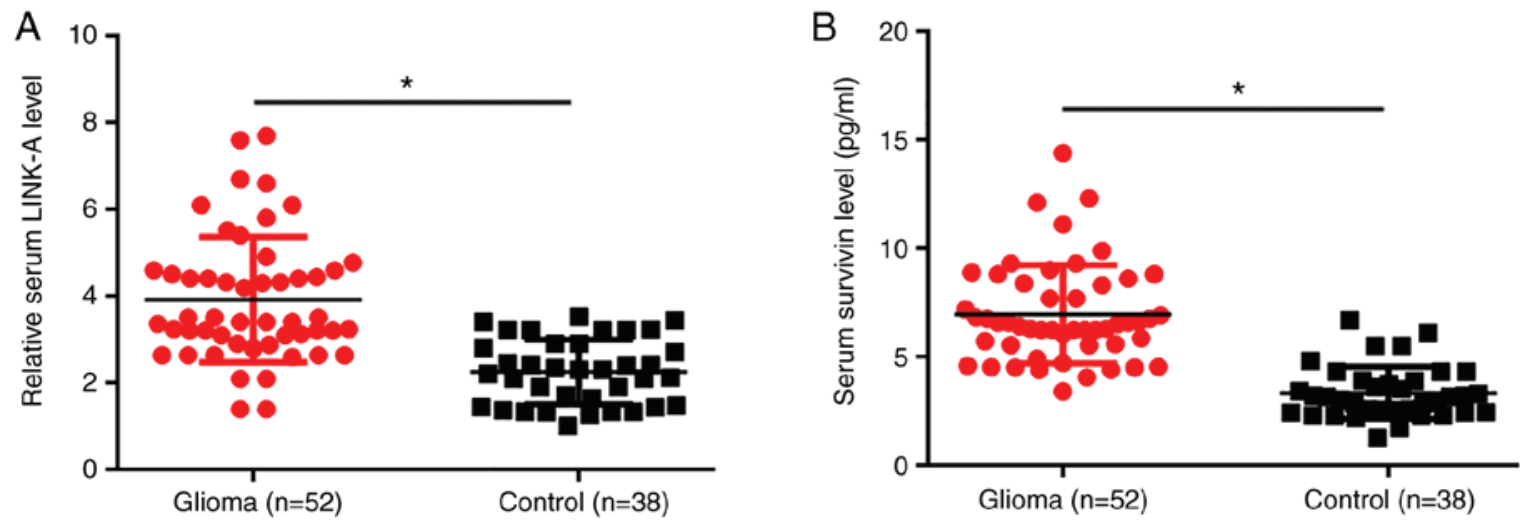

Figure 1. Serum levels of (A) LINK-A lncRNA and (B) survivin in patients with glioma and healthy controls. Compared with healthy controls, serum levels of LINK-A lncRNA and survivin were significantly increased in glioma patients. " $\mathrm{P}<0.05$. LINK-A, long intergenic non-coding RNA for kinase activation; lncRNA, long non-coding RNA.
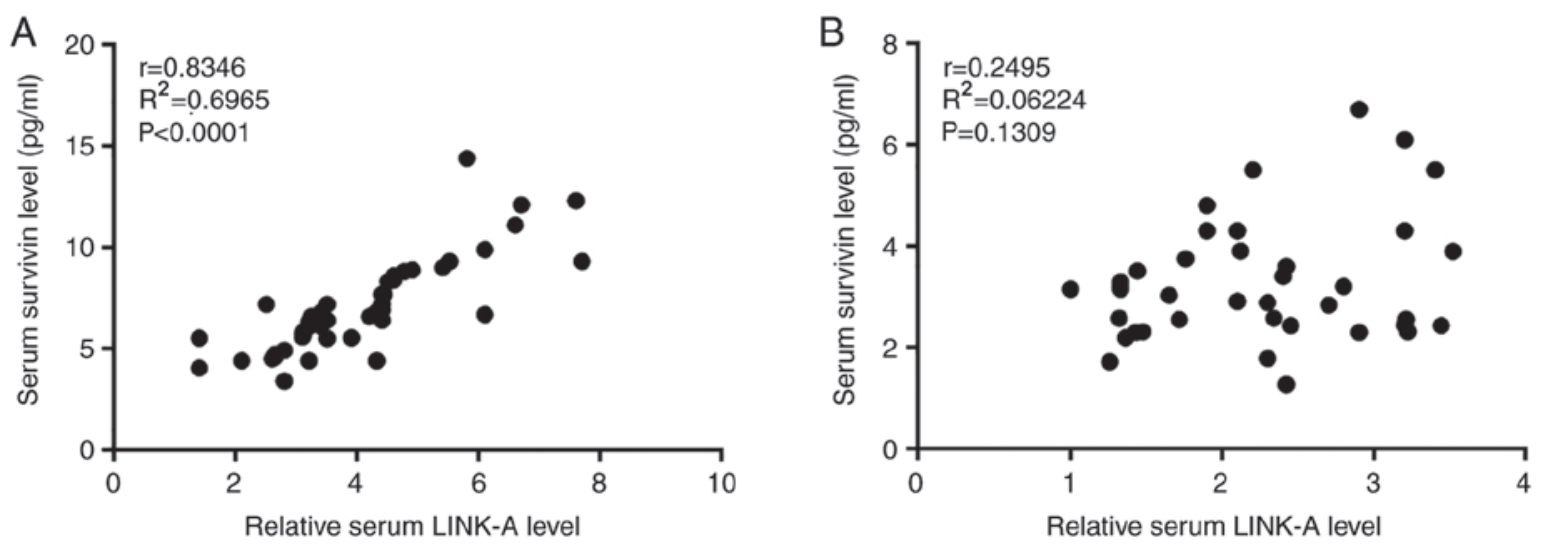

Figure 2. Correlation analysis of serum levels of LINK-A lncRNA and survivin in (A) patients with glioma and (B) healthy controls. Pearson's correlation coefficient revealed a significant positive correlation between serum levels of LINK-A lncRNA and survivin in glioma patients, but not in healthy controls. LINK-A, long intergenic non-coding RNA for kinase activation; lncRNA, long non-coding RNA.

in patients with glioma $(\mathrm{P}<0.05$; Fig. 1A). ELISA results showed that the serum level of survivin was also significantly higher in patients with glioma compared with healthy controls ( $\mathrm{P}<0.05$; Fig. 1B).

Serum levels of LINK-A IncRNA and survivin are positively correlated in patients with glioma. Serum levels of LINK-A lncRNA and survivin were analyzed using Pearson's correlation coefficient. As presented in Fig. 2A, a significant positive correlation between serum levels of LINK-A IncRNA and survivin was observed in patients with glioma. By contrast, no correlation was observed in healthy controls (Fig. 2B).

Increased serum levels of LINK-A IncRNA distinguish patients with glioma from healthy controls. The diagnostic value of serum LINK-1 lncRNA for glioma was evaluated by ROC curve analysis using glioma patients as true positive cases and healthy controls as true negative cases. As presented in Fig. 3, the area under the curve was 0.8543 (standard error, 0.0394; 95\% confidence interval, 0.7770-0.9315; $\mathrm{P}<0.001)$.

LINK-A IncRNA is an upstream activator of survivin in glioma cells. Confirmation of LINK-A overexpression and knockdown and survivin overexpression in CCD-25Lu and Hs 683

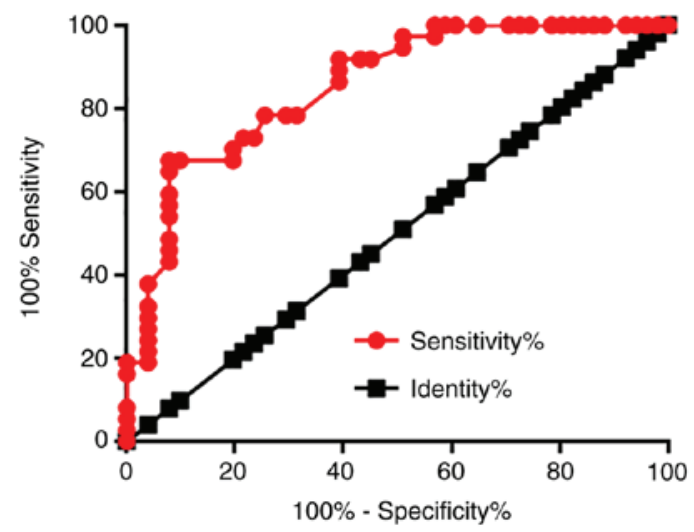

Figure 3. Receiver operating characteristic curve analysis of the diagnostic value of serum LINK-A lncRNA for glioma. Increased serum levels of LINK-A lncRNA distinguished patients with glioma from healthy controls. LINK-A, long intergenic non-coding RNA for kinase activation; lncRNA, long non-coding RNA.

human glioma cell lines following transfection is presented in Fig. $4(\mathrm{P}<0.05)$. After transfection, the expression levels of LINK-A IncRNA and survivin were detected by RT-qPCR and western blotting, respectively. Compared with the control 

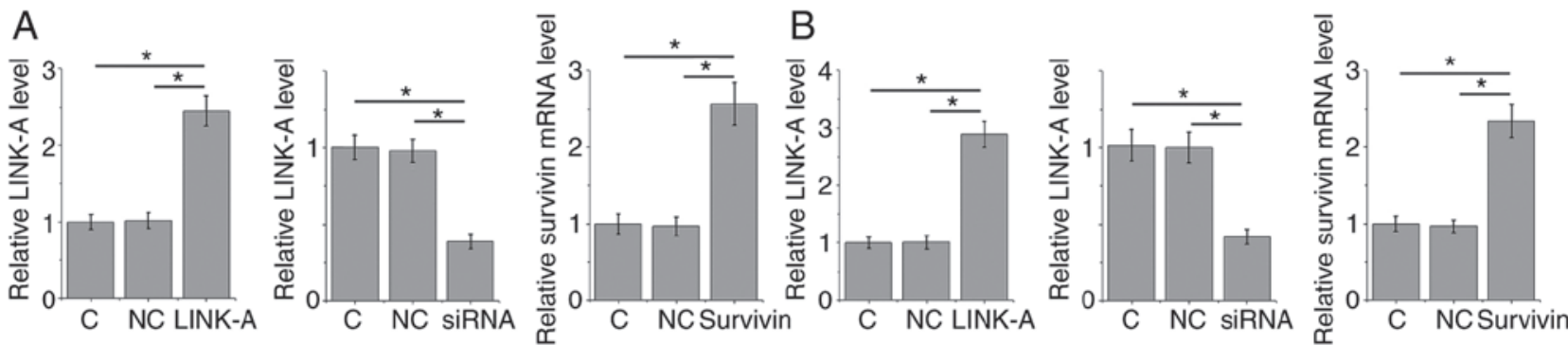

Figure 4. Confirmation of LINK-A overexpression and knockdown and survivin overexpression in (A) CCD-25Lu and (B) Hs 683 human glioma cell lines. ${ }^{*} \mathrm{P}<0.05$. LINK-A, long intergenic non-coding RNA for kinase activation; IncRNA, long non-coding RNA; C, control; NC, negative control; LINK-A, LINK-A overexpression group; Survivin, surviving overexpression group.

A
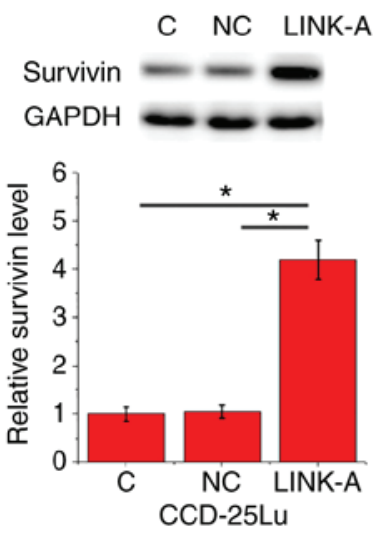

Hs 683

C NC LINK-A

\section{Survivin} GAPDH

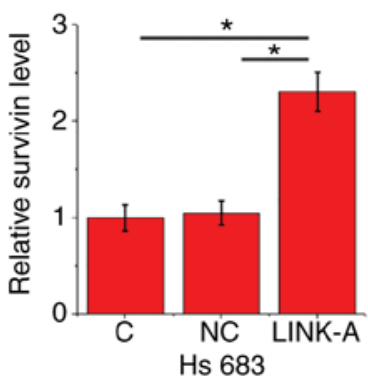

B

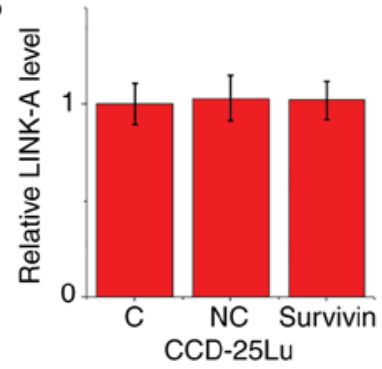

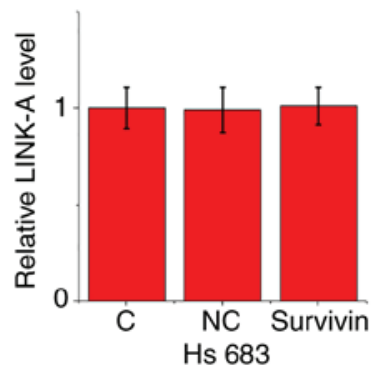

Figure 5. LINK-A lncRNA may be an upstream activator of survivin in CCD-25Lu and Hs 683 human glioma cell lines. (A) Western blot images and quantification of survivin in glioma cell lines transfected with LINK-A expression vector. (B) LINK-A lncRNA expression in glioma cell lines transfected with survivin expression vector. ${ }^{*} \mathrm{P}<0.05$. LINK-A, long intergenic non-coding RNA for kinase activation; IncRNA, long non-coding RNA; C, control; NC, negative control; LINK-A, LINK-A overexpression group; Survivin, survivin overexpression group.
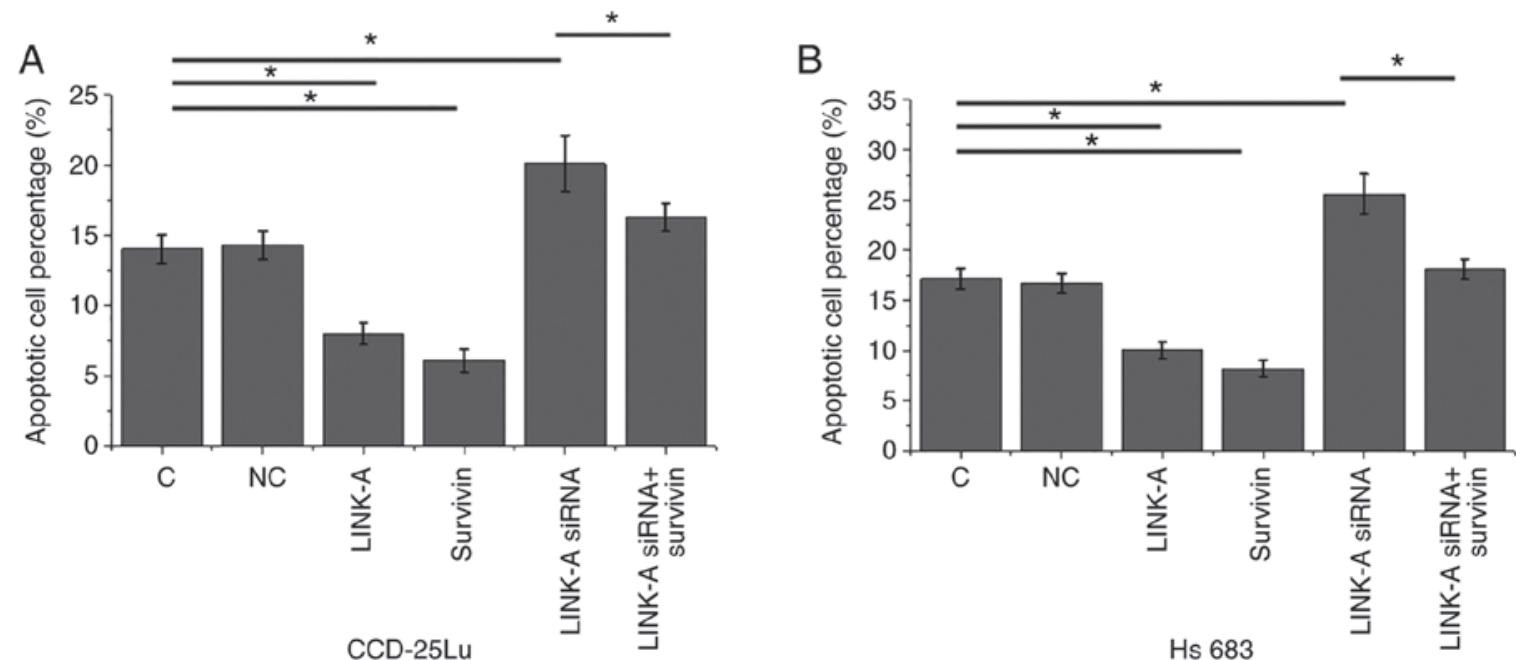

Figure 6. LINK-A lncRNA overexpression inhibits apoptosis in (A) CCD-25Lu and (B) Hs 683 human glioma cell lines, potentially via regulation of survivin. LINK-A IncRNA and survivin overexpression inhibited, while LINK-A lncRNA knockdown promoted the apoptosis of glioma cell lines. Survivin overexpression attenuated the inhibitory effects of LINK-A lncRNA knockdown on apoptosis. ${ }^{*} \mathrm{P}<0.05$. LINK-A, long intergenic non-coding RNA for kinase activation; lncRNA, long non-coding RNA; C, control; NC, negative control; LINK-A, LINK-A overexpression group; Survivin, survivin overexpression group.

and negative control groups, LINK-A lncRNA overexpression resulted in significantly upregulated expression of survivin in CCD-25Lu and Hs 683 human glioma cell lines $(\mathrm{P}<0.05$;
Fig. 5A). Compared with the control and negative control groups, survivin overexpression did not significantly affect LINK-A expression ( $\mathrm{P}<0.05$; Fig. 5B). 
LINK-A IncRNA overexpression inhibits apoptosis glioma cell through survivin. Cell apoptosis was detected following transfection. Compared with the control and negative control groups, LINK-A lncRNA and survivin overexpression significantly inhibited apoptosis in CCD-25Lu and Hs 683 human glioma cell lines ( $\mathrm{P}<0.05$; Fig. 6). The siRNA-mediated knockdown of LINK-A IncRNA resulted in significantly increased apoptosis of CCD-25Lu and Hs 683 glioma cell lines $(\mathrm{P}<0.05)$. In addition, survivin overexpression attenuated the inducing effect of LINK-A knockdown on glioma cell apoptosis $(\mathrm{P}<0.05)$.

\section{Discussion}

A recent study reported that LINK-A lncRNA serves a role in triple negative breast cancer as an oncogene by regulating energy metabolism (13). However, the involvement of LINK-A in other human diseases is unknown. In the present study, the role of LINK-A lncRNA in glioma as an oncogene was investigated. The effects of LINK-A lncRNA in glioma cells may be mediated through the upregulation of survivin, which is a key player in cancer development and progression (7-10).

Survivin has been found to be overexpressed during the development of different types of human cancers, suggesting it may serve a regulatory role in cancer cell apoptosis (7). In glioma, the overexpression of survivin has been associated with accelerated cancer cell proliferation and inhibited cancer cell apoptosis in glioma (15). Detection of survivin expression in tumor tissue is informative in estimating the prognosis of glioma patients (16). Survivin inhibitors are considered as potential therapeutic drugs for the treatment of human cancer, including glioma $(8,9,17)$. Consistent with previous studies $(8,9,17)$, the present study showed that the serum level of survivin was significantly higher in patients with glioma compared with healthy controls. In addition, the overexpression of survivin significantly inhibited the apoptosis of glioma cells in vitro. These data further confirm the oncogenic role of survivin in glioma.

LncRNAs serve important roles in glioma (18-21). Differentially expressed lncRNAs in glioma promote or inhibit cancer development $(18,19)$. In triple negative breast cancer, LINK-A lncRNA was found to be overexpressed, and potentially acts as an oncogene (13). In the present study, LINK-A lncRNA expression was observed to be significantly upregulated in patients with glioma compared with healthy controls. Additionally, upregulation of LINK-A in serum distinguished patients with glioma from healthy controls, indicating that it may have potential as a biomarker in the diagnosis of glioma. Additionally, LINK-A IncRNA overexpression inhibited, while siRNA-mediated knockdown of LINK-A promoted the apoptosis in cultured glioma cells in vitro. Therefore, inhibition of LINK-A IncRNA may serve as a potential therapeutic target for glioma.

A significant correlation was observed between the expression levels of LINK-A lncRNA and survivin in serum of patients with glioma indicating that there may be a potential interaction between them. In vitro experiments demonstrated that LINK-A IncRNA may be an upstream activator of survivin in the regulation of apoptosis in glioma cells. However, the mechanism of the role of LINK-A lncRNA in the regulation of survivin expression is unknown. Disease-related mediators may exist, as no correlation was observed between LINK-A IncRNA and survivin in the serum of healthy controls in the present study. It is known that LINK-A lncRNA promotes cancer development by activating normoxic HIFl $\alpha$ signaling in triple negative breast cancer (13). However, LINK-A lncRNA overexpression failed to significantly affect HIF1 $\alpha$ in glioma cells (data not shown). Since a previous study found that LINK-A IncRNA can interact with phosphatidylinositol-3,4,5-trisphosphate, which may also participate in cancer biology (22), future studies will focus on the interactions between LINK-A IncRNA and phosphatidylinositol-3,4,5-trisphosphate in glioma. A limitation of the present study is that it did not measure LINK-A lncRNA expression in tumor tissues; however, circulating RNA levels are able to reflect gene expression in tumors (23).

In conclusion, LINK-A IncRNA and survivin were upregulated in serum from patients with glioma. In addition, LINK-A lncRNA may inhibit apoptosis in glioma cells by upregulating survivin.

\section{Acknowledgements}

Not applicable.

\section{Funding}

No funding was received.

\section{Availability of data and materials}

The datasets used and/or analyzed during the present study are available from the corresponding author on reasonable request.

\section{Authors' contributions}

$\mathrm{XH}$ designed experiments. XH and GL performed experiments. $\mathrm{ZL}$ and $\mathrm{ZN}$ collected and analyzed data. $\mathrm{XH}$ drafted this manuscript. All authors read and approved this manuscript.

\section{Ethics approval and consent to participate}

The present study was approved by the Ethics Committee of the General Hospital of Ningxia Medical University. All patients and healthy volunteers provided written informed consent prior to their inclusion in the study.

\section{Patient consent for publication}

Not applicable.

\section{Competing interests}

The authors declare that they have no competing interests.

\section{References}

1. Goodenberger ML and Jenkins RB: Genetics of adult glioma. Cancer Genet 205: 613-621, 2012.

2. Mamelak AN and Jacoby DB: Targeted delivery of antitumoral therapy to glioma and other malignancies with synthetic chlorotoxin (TM-601). Expert Opin Drug Deliv 4: 175-186, 2007.

3. Macartney G, Harrison MB, VanDenKerkhof E, Stacey D and McCarthy P: Quality of life and symptoms in pediatric brain tumor survivors: A systematic review. J Pediatr Oncol Nurs 31: 65-77, 2014. 
4. Rice T, Lachance DH, Molinaro AM, Eckel-Passow JE, Walsh KM, Barnholtz-Sloan J, Ostrom QT, Francis SS, Wiemels J, Jenkins RB, et al: Understanding inherited genetic risk of adult glioma-a review. Neurooncol Pract 3: 10-16, 2016.

5. Radner H, el-Shabrawi Y, Eibl RH, Brüstle O, Kenner L, Kleihues $\mathrm{P}$ and Wiestler OD: Tumor induction by ras and myc oncogenes in fetal and neonatal brain: Modulating effects of developmental stage and retroviral dose. Acta Neuropathol 86: 456-465, 1993

6. Sah NK, Khan Z, Khan GJ and Bisen PS: Structural, functional and therapeutic biology of survivin. Cancer Lett 244: 164-171, 2006.

7. Jaiswal PK, Goel A and Mittal RD: Survivin: A molecular biomarker in cancer. Indian J Med Res 141: 389-397, 2015.

8. Chen X, Duan N, Zhang C and Zhang W: Survivin and tumorigenesis: Molecular mechanisms and therapeutic strategies. J Cancer 7: 314-323, 2016.

9. Garg H, Suri P, Gupta JC, Talwar GP and Dubey S: Survivin: A unique target for tumor therapy. Cancer Cell Int 16: 49, 2016.

10. Mobahat M, Narendran A and Riabowol K: Survivin as a preferential target for cancer therapy. Int J Mol Sci 15: 2494-2516, 2014.

11. Chen H, Yang F, Li X, Gong ZJ and Wang LW: Long noncoding RNA LNC473 inhibits the ubiquitination of survivin via association with USP9X and enhances cell proliferation and invasion in hepatocellular carcinoma cells. Biochem Biophys Res Commun 499: 702-710, 2018.

12. Yang R, Qu S, Liang H, Chen X, Zhang C and Guo H: Long noncoding RNA H19 regulates survivin expression in bladder cancer as sponge of miR-138-5p. Eur Urol Suppl 16: e1464-e1465, 2017.

13. Lin A, Li C, Xing Z, Hu Q, Liang K, Han L, Wang C, Hawke DH, Wang S, Zhang Y, et al: The LINK-A IncRNA activates normoxic HIF1 $\alpha$ signalling in triple-negative breast cancer. Nat Cell Biol 18: 213-224, 2016.

14. Livak KJ and Schmittgen TD: Analysis of relative gene expression data using real-time quantitative PCR and the 2(-Delta Delta C(T)) method. Methods 25: 402-408, 2001.
15. Zhang F, Chu J and Wang F: Expression and clinical significance of cyclooxygenase 2 and survivin in human gliomas. Oncol Lett 14: 1303-1308, 2017

16. Lv S, Dai C, Liu Y, Shi R, Tang Z, Han M, Bian R, Sun B and Wang R: Retraction note to: The impact of survivin on prognosis and clinicopathology of glioma patients: A systematic meta-analysis. Mol Neurobiol 54: 2376, 2017.

17. Jane EP, Premkumar DR, Sutera PA, Cavaleri JM and Pollack IF: Survivin inhibitor YM155 induces mitochondrial dysfunction autophagy,DNA damage and apoptosisin Bcl-xL silenced glioma cell lines. Mol Carcinog 56: 1251-1265, 2017.

18. Zhang X, Sun S, Pu JK, Tsang AC, Lee D, Man VO, Lui WM, Wong ST and Leung GK: Long non-coding RNA expression profiles predict clinical phenotypes in glioma. Neurobiol Dis 48: 1-8, 2012.

19. Wang P, Ren Z and Sun P: Overexpression of the long non-coding RNA MEG3 impairs in vitro glioma cell proliferation. J Cell Biochem 113: 1868-1874, 2012.

20. Zhang H, Wei DL, Wan L, Yan SF and Sun YH: Highly expressed lncRNA CCND2-AS1 promotes glioma cell proliferation through Wnt $/ \beta$-catenin signaling. Biochem Biophys Res Commun 482: 1219-1225, 2017.

21. Liao Y, Shen L, Zhao H, Liu Q, Fu J, Guo Y, Peng R and Cheng L: LncRNA CASC2 interacts with miR-181a to modulate glioma growth and resistance to TMZ through PTEN pathway. J Cell Biochem 118: 1889-1899, 2017.

22. Lin A, Hu Q, Li C, Xing Z, Ma G, Wang C, Li J, Ye Y, Yao J, Liang K, et al: The LINK-A lncRNA interacts with PtdIns $(3,4,5)$ $\mathrm{P}_{3}$ to hyperactivate AKT and confer resistance to AKT inhibitors. Nat Cell Biol 19: 238-251, 2017.

23. Kopreski MS, Benko FA, Kwak LW and Gocke CD: Detection of tumor messenger RNA in the serum of patients with malignant melanoma. Clin Cancer Res 5: 1961-1965, 1999.

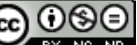

This work is licensed under a Creative Commons Attribution-NonCommercial-NoDerivatives 4.0 International (CC BY-NC-ND 4.0) License. 